\title{
A short review about some exotic systems containing electrons, muons, and tauons
}

\author{
Mohsen Emami-Razavi
}

Received: 16 August 2014/ Accepted: 27 October 2014/Published online: 10 December 2014

(C) The Author(s) 2014. This article is published with open access at Springerlink.com

\begin{abstract}
Some remarks about exotic systems consisting of various masses of fermions and antifermions have been presented. In paricular, few body systems containing electrons, muons and tauons, and the fundamental interest of the study of their bound states systems have been discussed.
\end{abstract}

Keywords Exotic Systems · Muons - Tauons ·

True Muonium · Bound states

\section{Introduction}

The purpose of the present paper is to present some remarks about the importance of the properties of exotic systems for arbitrary fermions and antifermions. We discuss also the bound states of the systems of two or more than two fermionantifermion that include "muons" and "tauons" (systems like $\left.\mu^{+} \tau^{-}, e^{-} \tau^{-} \mu^{+}, e^{-} \mu^{+} e^{-} \mu^{+}\right)$. The investigation of the stability of some exotic systems, bound states energies and the properties of three-, four-, and five-body systems that contain muons and/or tauons is of fundamental interest in Quantum Electrodynamics (QED).

In a paper [1], it has been shown that the production and study of true muonium is possible at modern electronpositron colliders. The true muonium $\left(\mu^{+} \mu^{-}\right)$, true tauonium $\left(\tau^{+} \tau^{-}\right)$, and "mu-tauonium" $\left(\mu^{ \pm} \tau^{\mp}\right)$ bound states are not only the heaviest, but also the most compact QED systems. Hence, from fundamental point of view the study of the bound states systems that contain muons and tauons

M. Emami-Razavi (凶)

Plasma Physics Research Center, Science and Research Branch, Islamic Azad University, P.O. Box 14665-678, Tehran, Iran

e-mail: mrazavi@srbiau.ac.ir is of interest. The rapid weak decay of the $\tau$ makes the observation of the systems such as $\left(\tau^{+} \tau^{-}\right)$or $\left(\mu^{ \pm} \tau^{\mp}\right)$ difficult.

A muon is a particle which has similar properties as the electron, except that it is about 207 times heavier than the electron $\left(m_{\mu} \simeq 207 m_{e}\right)$. Muon has a lifetime of around $2.2 \times 10^{-6}$ second. Some effects, which play only a minor role for the electron in usual atoms, become important for the $\mu^{-}$(or the $\mu^{+}$) when it circles around the nucleus forming a muonic atom $[2,3]$. These effects are linked to the large mass of the muon, which implies that the Bohr radius of the $\mu^{-}$is smaller than of the electron by a factor of $\frac{1}{127}$. Similarly, the tauon $\left(\tau^{-}\right)$is about 3,477 times heavier than the electron and since its interaction is very similar to that of the electron, a tauon can be thought of as a much heavier version of the electron. Even though a tauon has similar properties as the electron, its short lifetime (about $2.9 \times 10^{-13} \mathrm{~s}$ ) makes it much more difficult than a muon to study and thereby to obtain experimental results.

The simplest few-body problem for a system of fermions and antifermions of equal masses with electromagnetic interactions is that of positronium (Ps: $e^{-} e^{+}$). Deutsch [4] was the first person who observed Ps in 1951. Since the discovery of Ps, there have been major advances in understanding of the Ps system and in the use of Ps to explore the basic structure of QED [5, 6]. Theoretical studies of the Ps system are now well advanced. For example, accurate calculations of the positronium hyperfine interval contributions at the level of $O\left(\alpha^{6}\right)$ ground-state hyperfine splitting in positronium have been studied by Adkins et al. [5,6]. An account of the history of QED has been written by Schweber [7] (see also Dyson [8]). 
Positronium plays a unique role in the continuing development of the techniques of bound-state QED and high-precision tests of the standard model as a Coulombic bound system that is almost free of strong and weak interaction contaminations and that exhibits large recoil and annihilation (virtual and real) effects. In very recent works, Adkins et al. [9, 10] calculated the positronium hyperfine splitting and energy levels at order $O\left(\alpha^{7}\right)$. They obtained a new and more precise result for the light-bylight scattering correction to the real decay of parapositronium into two photons. Adkins et al. have also calculated the three-loop correction to the positronium hyperfine splitting due to light-by-light scattering in the exchange of two photons between the electron and positron.

The positronium negative ion $\left(\mathrm{Ps}^{-}\right)$, composed of three equal mass fermions $\left(e^{+} e^{-} e^{-}\right)$, is the simplest three-body system bound only by electromagnetic interactions. The existence of a bound $\mathrm{Ps}^{-}$system was predicted by Wheeler [11]. Theoretical studies of the $\mathrm{Ps}^{-}$are also well advanced (see, for example, Drake and Grigorescu [12] or Frolov [13]). The Ps ${ }^{-}$system was first observed by Mills [14], and later on by Fleischer et al. [15].

With respect to the relativistic and QED corrections for the positronium negative ion system, we can quote the following example. In Ref. [12], the leading relativistic and QED corrections to the ground-state energy of the threebody system $\left(e^{+} e^{-} e^{-}\right)$have been calculated numerically using a Hylleraas correlated basis set. The accuracy of the non-relativistic variational ground state in [12] is discussed with respect to the convergence of the energy with increasing size of the basis set, and also with respect to the variance of the Hamiltonian. The corrections to this energy include the lowest order Breit interaction, the vacuum polarization potential, one and two photon exchange contributions, the annihilation interaction and spin-spin contact terms.

Fundamental fermions antifermions systems with electromagnetic interactions are of interest because they are "pure" QED systems, with point-like constituents and no nuclear force or size effects. Experiments on such "exotic" atoms, though difficult, are being undertaken not only for positronium and the three-body $\mathrm{Ps}^{-}\left(e^{+} e^{-} e^{-}\right)$system, but also for the four-body "positronium molecule" $\left(\mathrm{Ps}_{2}\right.$ : $\left.e^{+} e^{-} e^{+} e^{-}\right)$. The positronium molecule was observed in 2007 by Cassidy and Mills [16]. The existence of a boundstate $\mathrm{Ps}_{2}$ was first predicted by Hylleraas and Ore [17]. There are many papers on this topic in the literature (see, for example, Emami-Razavi [18] for a review of earlier work). Moreover, the dipole excitation of the positronium molecule (the energy interval between ground and the $P$ wave exited state of $\mathrm{Ps}_{2}$ ) and thermal instability of $\mathrm{Ps}_{2}$ have been recently studied, respectively, in [19] and [20].
For the positronium molecule system Bubin et al. [21] solved the non-relativistic problem variationally and used their solutions to calculate the relativistic corrections in first-order perturbation theory. Their result for the groundstate binding energy of $\mathrm{Ps}_{2}$ is 0.01595425 Hartree (i.e., $0.4341373 \mathrm{eV})$. This includes the $O\left(\alpha^{2}\right)$ relativistic corrections to the non-relativistic ground-state energy of $\mathrm{Ps}_{2}$ system.

The simplest system for fermions and antifermions of different flavor is muonium, $e^{-} \mu^{+}$. This system (and muonium-like systems) have been investigated in the literature (see, for example, [22] and refs therein). The term "muonium" for the $e^{-} \mu^{+}$bound state and its first theoretical discussion appeared in Ref. [23], and the state was discovered soon thereafter [24].

For a three-body system of fermions and antifermions of various flavors, we can mention, for example, the system consisting of two identical particles and a different antiparticle (e.g., muonium negative ion: $\mathrm{Mu}^{-}$or $e^{-}, e^{-}, \mu^{+}$). The first observation of the negative muonium ion produced by electron capture in a beam-foil experiment has been done by Kuang et al. [25]. This system has been studied, for example, by Frolov [26], and by Barham and Darewych [27]. The $\mathrm{Mu}^{-}$has only one bound (ground) state ${ }^{1} S(L=0)$ state [28]. The energies and other boundstate properties for the ground ${ }^{1} S(L=0)$ state in the $\mathrm{Mu}^{-}$ ion are known to very high accuracy [29].

The four-body system $\left(m^{Z^{+}}, \mathrm{Ps}^{-}\right), m$ is the mass of the particle to be specified with respect to $\mathrm{Ps}^{-}$and $Z$ is the number of the charge has been studied in Ref. [30]. The properties of some exotic five-particle systems have been studied in [31]. A proof of stability of four-body system, hydrogen, hydrogen-like molecules $\left(M^{+} M^{+} m^{-} m^{-}\right)$, and some asymmetric molecules of the type $\left(m_{1}^{-}, m_{2}^{+}, m_{3}^{-}\right.$, $m_{4}^{+}$) has been discussed by Richard [32] (see also [33]).

The prediction of the stability of Coulombic few-body systems requires sophisticated calculations [34]. As pointed out, for example in Ref. [31], the difficulty can largely be attributed to the fact that the correlations between like and opposite charges are quite different due to the attractive and repulsive interaction. Another factor which plays a crucial role in the binding mechanism for fermions and antifermions is the Pauli principle. The main forces to determine the stability domains are the Pauli principle and the mass ratios [31]. The Pauli principle severely restricts the available configuration space for fermionic systems.

As far as the two-body system interacting via Coulombic force is concerned, it is possible to find the binding energy of the systems analytically and there are many papers available in the literature. However, when one has a system of three- or four-body system, it is not possible to obtain analytical solution for the binding energy of the 
systems. Therefore, one needs to perform some numerical calculations. Those computations of stochastic variational method (SVM) or a variant of this method have been presented in $[35,37]$. In these work, the diatomic basis sets were used to calculate energies or other properties of the exotic systems under study [37].

The presentation of this work is as following. The two-, three-, four- and five-body systems are discussed from section two to section five. Concluding remarks are included in the last section.

\section{Two-body systems}

Consider a system of $N$ particles with masses $\left(m_{1}, m_{2}, \ldots\right.$, $m_{N}$, and charges $\left(q_{1}, q_{2}, \ldots, q_{N}\right)$. If we take the particles to be charged point masses with Coulombic interactions, then the non-relativistic Hamiltonian has the familiar form

$H=-\sum_{i=1}^{N} \frac{\hbar^{2}}{2 m_{i}} \nabla_{i}^{2}+\sum_{i<j}^{N} \frac{q_{i} q_{j}}{\left|\mathbf{r}_{\mathbf{i}}-\mathbf{r}_{\mathbf{j}}\right|}$.

The simplest few body exotic systems corresponds to the two-body case and analytical solutions can be obtained for the binding energy of a two-body system. However for a three-body system (or four-body system) numerical calculations are needed to obtain numerical results [36, 37].

The simplest two-body systems that contain muons are the true muonium $\mu^{+} \mu^{-}$and muonium $e^{-} \mu^{+}$(discovered by Hughes [24]). Due to the close confinement in the bound state muonium can be used as an ideal probe of electroweak interaction, including particularly QED, and to search for additional yet unknown interactions acting on leptons. The term "muonium" for the $e^{-} \mu^{+}$bound state and its first theoretical discussion appeared in Ref. [23], and the state was discovered soon thereafter. Since then, this system (and muonium-like systems) have been investigated in the literature by many authors, particularly for the nonrelativistic case. For the relativistic and QED corrections of the muonium-like systems, there are some papers available in the literature (see, for example, [22] and refs therein).

Terekidi and Darewych [22] have considered a reformulation of QED in which covariant Green functions are used to solve for the electromagnetic field in terms of the fermion fields. The resulting modified Hamiltonian contains the photon propagator directly. The authors [22] obtained solutions of the two-body equations for muoniumlike system to the order of $O\left(\alpha^{4}\right)$. Their results compare well with the observed muonium spectrum, as well as that for hydrogen and muonic hydrogen. Anomalous magnetic moment effects are also discussed in [22].

Unlike in the case of positronium, the true muonium $\left(\mu^{+} \mu^{-}\right)$constituents themselves are unstable. However, the $\mu$ has an exceptionally long lifetime by particle physics standards $\left(2.2 \times 10^{-6}\right.$ second $)$, meaning that $\left(\mu^{+} \mu^{-}\right)$ annihilates long before its constituents weakly decay. Hence, the $\mu^{+} \mu^{-}$is unique as the heaviest metastable laboratory possible test for precision QED tests. It has a lifetime of $0.602 \mathrm{Ps}$ in the ${ }^{1} S_{0}$ state (decaying to $\gamma \gamma$ ) and $1.81 \mathrm{ps}$ in the ${ }^{3} S_{1}$ state (decaying to $e^{+} e^{-}$) [38-39]. One should note those systems are the most compact two-body QED systems and therefore from fundamental point of view their studies are of interest.

In principle, the creation of true tauonium $\left(\tau^{+} \tau^{-}\right)$and "mu-tauonium" $\left(\mu^{ \pm} \tau^{\mp}\right)$ are also possible but the relatively short lifetime of tauon makes it difficult to observe such systems with current experimental setups. The corresponding ${ }^{1} S_{0}$ and ${ }^{3} S_{1}$ lifetimes of $\left(\tau^{+} \tau^{-}\right)$are 35.8 and 107 fs [1], respectively, to be compared with the free $\tau$ lifetime $291 \mathrm{fs}$ (or half this for a system of two $\tau^{\prime}$ s). The $\left(\tau^{+} \tau^{-}\right)$ annihilation decay and the weak decay of the constituent $\tau^{\prime}$ 's actually compete, making $\left(\tau^{+} \tau^{-}\right)$not a pure QED system like $\left(e^{+} e^{-}\right)[1]$.

One should note that due to very short lifetime of tauon, the systems such as $\left(\mu^{+} \tau^{-}\right)$have a very short lifetime and they are not true bound states; in other words, they constitute quasi-bound states (or resonances) and it is highly unlikely that they will be observed in near future experiments.

\section{Three-body systems}

The three-body bound states with electromagnetic interaction have been studied since many years ago by several authors (see, for example, Bhatia and Drachman [40]). A review of some particular three unit charge systems along with the domain of stability of those systems has been presented, for example in Ref. [33].

The positronium negative ion $\left(\mathrm{Ps}^{-}\right)$, composed of three equal mass fermions $\left(e^{+} e^{-} e^{-}\right)$, is the simplest three-body system with Coulombic interaction. The other three-body system of interest is muonium negative ions $\left(\mu^{+} e^{-} e^{-}\right)$. Note that for muonium negative ion $\left(\mathrm{Mu}^{-}: \mu^{+} e^{-} e^{-}\right)$, the muonium conversion (or $\mu^{+} e^{-} \longrightarrow \mu^{-} e^{+}$conversion) in the $\mathrm{Mu}^{-}$ion can produce a number of secondary atomic processes and it is the most interesting process which can be observed with $\mu^{+}$muons in atomic structures [26]. In fact, $\mu^{+} e^{-} \longrightarrow \mu^{-} e^{+}$conversion transforms the incident three-body system $\left(\mu^{+} e^{-} e^{-}\right)$into a system $\left(\mu^{-} e^{+} e^{-}\right)$ which has no bound state [28]. The theoretical investigation of those systems are now well advanced (relativistic corrections of $\mathrm{H}^{-}$, positronium and muonium negative ions have been studied, for example, by Barham and Darewych, see [27] and references therein). 
In [27], the authors discussed relativistic three-fermion wave equations in QED. The equations are used to obtain relativistic $O\left(\alpha^{2}\right)$ corrections to the non-relativistic groundstate energy levels of the positronium and muonium negative ions, as well as $\mathrm{H}^{-}$, using approximate variational three-body wavefunctions. The results of [27] have been compared with other calculations, where available.

In a paper by Frolov [41] using the approach of explicitly correlated exponential basis functions has studied the properties of some atomic and molecular three-body ions which contain positively charged muons $\left(\mu^{+}\right)$. In particular, the ground states in the muonium ion $\left(\mathrm{Mu}^{-}\right.$: $\left.\mu^{+} e^{-} e^{-}\right)$and muon-hydrogen molecular $\left(p^{+} \mu^{+} e^{-}\right)$, $\left(d^{+} \mu^{+} e^{-}\right)$, and $\left(t^{+} \mu^{+} e^{-}\right)$ions $(d, t$ stand for deuteron and tritium) have been examined in details and the energies of these systems have been determined to high accuracy.

We remind again that due to short lifetimes of $\mu$ and $\tau$, these systems are quasi-bound state and very difficult to detect by experimental equipments of nowadays. However, from theoretical point of view it would be of interest to see that some of these systems cannot even be bound and some of them can be stable enough to constitute a bound state.

\section{Four-body systems}

The solution of the four-body system is a very difficult problem. However, despite the complexity of the calculations, enormous progress has been made (see for, example [33], for a review) in this field which has been rapidly developing ever since of the birth of non-relativistic quantum mechanics (Schrödinger equation). Moreover, one should note that the QED and relativistic corrections of the four-body systems of different flavors still remain a very challenging problem. However, in a recent paper [42], the relativistic wave equations of systems consisting of fermions and antifermions of various masses have been presented. To our knowledge, there are no major work or study for the "relativistic" or QED calculations of fourbody system of different masses.

The theoretical investigation of the "non-relativistic" four-body systems interacting through Coulombic potential has been discussed in several works and some studies have been done regarding the existence of bound states of those systems and also the properties of the domain of stability in the space masses or inverse masses [33] (those results are supplemented by numerical investigations using accurate variational methods). The stability domain of the system made up of four particles of different masses, two having the same positive charge and two having the opposite negative charge, interacting only through Coulombic forces, can be represented in terms of points in the interior, or on the surface of a regular tetrahedron [32].

Recent success in the production of trapped antihdyrogen atoms [43, 44] has renewed interest in the interaction of hydrogen-antihydrogen system. $H-\bar{H}$ is known to decay into protonium ( $p \bar{p}$, proton or antiproton is considered fundamental particle here) and positronium $\left(e^{+} e^{-}\right)$. In Ref. [45] it was pointed out that from unstable $H-\bar{H}$ system, it also follows that the systems $p \mu^{-} e^{+} e^{-}$, $\mu^{+} \mu^{-} e^{+} e^{-}, d \bar{p} e^{+} e^{-}$, and $t \bar{p} e^{+} e^{-}(d, t$ stand for deuteron and tritium) are unstable.

One of the system of interest consisting of fundamental particles is muonium molecule $\left(\mathrm{Mu}_{2}, e^{-} \mu^{+} e^{-} \mu^{+}\right.$: system consisting of two electrons and two antimuons). The only exotic QED four-body system consisting of fundamental particles that has been observed is the positronium molecule [16]. $\mathrm{Mu}_{2}$ system is not observed as yet. Some other exotic four-body systems are such as $e^{-} \mu^{+} e^{-} \mu^{+}$, $e^{-} \mu^{+} e^{-} p, e^{-} \mu^{+} e^{-} \tau^{+}$, and $e^{-} \mu^{+} \tau^{-} p$.

Bubin et al. [21] reported that they have obtained a very accurate variational wave function for non-relativistic binding energy of the positronium molecule $\left(\mathrm{Ps}_{2}\right)$, which they used to calculate the relativistic corrections. However, to our knowledge there is no study of relativistic or QED corrections for the four-body exotic systems for various masses of fermions and antifermions and it remains an open problem.

One should also note the following points about muonium molecule $\left(\mathrm{Mu}_{2}, e^{-} \mu^{+} e^{-} \mu^{+}\right)$in case of its existence. The muonium molecule system is $\mathrm{H}_{2}$ (hydrogen molecule) like. There is no virtual annihilation in the $\mathrm{Mu}_{2}$ and in this case it is very different from $\left(\mu^{+} \mu^{-} e^{+} e^{-}\right)$ system. In the latter system, there are two different virtual annihilation interactions, namely, $\left(\mu^{+} \mu^{-}\right)$and $\left(e^{+} e^{-}\right)$. The system $\left(\mu^{+} \mu^{-} e^{+} e^{-}\right)$is hydrogen-antihydrogen like and it is not bound according to Ref. [45].

Moreover, as it was pointed out for the three-body case, the muonium conversion (or $\mu^{+} e^{-} \longrightarrow \mu^{-} e^{+}$conversion) in the muonium molecule can transform the four-body system $\left(e^{-} \mu^{+} e^{-} \mu^{+}\right)$into the system $\left(\mu^{+} \mu^{-} e^{+} e^{-}\right)$, which has no bound state [45]. We remind that for the three-body case we mentioned earlier that the system $\left(\mu^{-} e^{+} e^{-}\right)$has no bound state [28], on the contrary to muonium negative ion $\left(\mu^{+} e^{-} e^{-}\right)$, which has a bound state [26], and it was observed by Kuang et al. [25]. In case of a future observation of muonium molecule $\left(e^{-} \mu^{+} e^{-} \mu^{+}\right)$, the comparison of recent observed positronium molecule $\left(e^{-} e^{+} e^{-} e^{+}\right)$ [16] with muonium molecule may provide a better understanding of theory of QED. 


\section{Five-body systems}

The study of exotic five-particle systems and the prediction of their stability requires very sophisticated calculations. Mezei et al. [31] did investigate the stability of a number of five-body systems using SVM. The small loosely bound systems require very accurate calculations. The properties of the most intriguing systems consisting of two electrons and two positrons (e.g., $e^{+} \mathrm{PsH}$ or $\mathrm{Li}^{+} \mathrm{Ps}_{2}$ ) have been investigated in great detail [31] for the non-relativistic Schrödinger equation.

The difficulty of the five-body calculations can largely be attributed to the fact that the correlations between like and opposite charges are quite different due to the attractive and repulsive interaction. Moreover, the Pauli principle plays a crucial role in the binding mechanism of the fermions. For systems with identical particles, the antisymmetry requirement seriously restricts phase space accessible to the particles by not allowing the energetically most favorable configurations. Furthermore, the total charges of a bound five particle system must be \pm 1 , that is, there is no bound system with $(+,-,-,-,-)$ charges [31].

The simplest five-body particles is a system consisting of three positively charged particles and two negatively charged identical fermions with spin $1 / 2 \mathrm{Cm}^{+} \mathrm{m}^{+} \mathrm{m}^{+}$ $m^{-} m^{-}$). The antisymmetry requirement restricts the configuration space and no bound state exists; and in particular, the system of three electrons and two positrons, is not bound [31]. However, the system $\left(m^{+} m^{+} m^{+} m^{-} m^{-}\right)$is bound if the three positively charged are bosons or if one of them is distinguishable, since the Pauli principle does not restrict the allowed states. An example is $\left(e^{+} e^{+} e^{+} e^{-} x^{-}\right)$ system, where $x^{-}$is a fictitious particle which has the same mass of the electron (or it can have different mass) but it is distinguishable from both electron and the positron [31]. For example, a system made of positronium molecule and a proton $\left(e^{+} e^{+} e^{-} e^{-} p\right)$ is stable. The properties of some other exotic five-particle systems $\left(m_{1}^{+} m_{2}^{+} m_{3}^{+} m_{4}^{-} m_{5}^{-}\right)$ where two or three of the particles may have the same mass have been discussed in [31] (see also [33] for a review).

On a practical level, it is very difficult to observe the five-body exotic systems that contains muon (or tauon) and electrons (for example: $e^{+} e^{+} e^{-} e^{-} \mu^{+}$). So far, to our knowledge the only exotic system that contains fundamental particles and antiparticles with electromagnetic interaction and has been observed is the positronium molecule $\left(e^{+} e^{+} e^{-} e^{-}\right)$[16]. However, from theoretical point of view, one should consider that the study of the exotic five-body systems and their application may be of interest [31] even though their observations will not be in the near future.

\section{Concluding remarks}

In this paper, we were particularly interested in presenting the study of the two-, three-, four-, and five-body exotic systems that contains electrons, muons, and tauons (with their corresponding antiparticles). The non-relativistic energies and bound states of those systems have been investigated by several authors. The relativistic or QED corrections of the energies of more than two-body exotic systems have not been considered by many authors and therefore remain a challenging problem.

For the two-body relativistic or QED corrections, such as for the positronium system, some advanced studies have recently been performed $[9,10]$ to calculate the positronium hyperfine splitting and energy levels at order $O\left(\alpha^{7}\right)$. Similarly, the relativistic or QED corrections for some particular three- and four-body systems have been investigated in various papers (see for examples, [12] and [21]).

The observation of positronium molecule by Cassidy and Mills [16] raises interest in other exotic systems. To our knowledge, there are not many papers in the literature that study the bound states of systems that contain a few muons and/or tauons combined with other particles such as $e^{-}$or $e^{+}$. Hence, at least from theoretical point of view, the study of such exotic systems would be of fundamental importance.

In a recent paper [46], the production and discovery of "True Muonium" (a bound state of a muon and antimuon) in fixed-target experiments have been discussed. According to the authors in Ref. [46], discovery and measurement prospects appear very favorable for the true muonium system.

To conclude, we can mention the following point. Experiments on "exotic" atoms or molecules, though difficult, will be undertaken in future even though it may not be near future. With the current experimental setup available at this time, it is expected the true muonium $\left(\mu^{+} \mu^{-}\right)$ system can be observed in near future (see, for example, [1] or [46]). Therefore, further test of QED systems and their corresponding theories can be achieved using new observed systems.

Acknowledgments The author thanks Professors Jim Mitroy and Jurij W. Darewych for useful comments.

Open Access This article is distributed under the terms of the Creative Commons Attribution License which permits any use, distribution, and reproduction in any medium, provided the original author(s) and the source are credited.

\section{References}

1. Brodsky, S.J., Lebed, R.F.: Phys. Rev. Lett. 102, 213401 (2009) 
2. Greiner, W.: Relativ. Quantum. Mech. Ch 9, p193, SpringerVerlag (1989)

3. Greiner W., Reinhardt, J.: Quantum. Electrodyn. Ch 5, p 200, Springer-Verlag (1989)

4. Deutsch, M.: Phys. Rev. 82, 455 (1951)

5. Adkins, G.S., Fell, R.N., Mitrikov, P.M.: Phys. Rev. Lett. 79, 3383 (1997)

6. Adkins, G.S., Fell, R.N., MitrikovPhys, P.M.: Rev. A 65, 042103 (2002)

7. Schweber, S.S.: QED and the Men who Made It: 1) Freeman Dyson, 2) Richard Feynman, 3) Julian Schwinger, and 4) Sin-Itiro Tomonaga. Princeton University Press, Princeton (1994)

8. Freeman, D., Adv. Quantum. Mechan. World. Sci. (2007)

9. Adkins, G.S., Parsons, C., Salinger, M.D., Wang, R., Fell, R.N.: Phys. Rev. A 90, 042502 (2014)

10. Adkins, G.S., Fell, R.N.: Phys. Rev. A 89, 052518 (2014)

11. Wheeler, J.A.: Ann. NY Acad. Sci. 48, 219 (1946)

12. Drake, G.W.F., Grigorescu, M.: J. Phys. B: At. Mol. Opt. Phys. 38, 3377-3393 (2005)

13. Frolov, A.M.: Phys. Lett. A 342, 430 (2005). Phys. Rev. A 60, 2834 (1999)

14. Mills Jr, A.P.: Phys. Rev. Lett. 46, 717 (1981). Phys. Rev. Lett. 50, 671 (1983)

15. Fleischer, F., et al.: Phys. Rev. Lett. 96, 063401 (2006)

16. Cassidy D.B., Mills A.P., Nature (London) 449

17. Hylleraas, E.A., Ore, A.: Phys. Rev. 71, 493 (1947)

18. Emami-Razavi, M.: Phys. Rev. A 77, 042104 (2008)

19. Puchalski, M., Czarnecki, A.: Phys. Rev. Lett. 101, 183001 (2008)

20. Kylänpää, I., Rantala, T.T, Phys. Rev. A 80, 024504 (2009).

21. Bubin, S., Stanke, M., Kedziera, D., Adamowicz, L.: Phys. Rev. A 75, 062504 (2007)

22. Terekidi, A.G., Darewych, J.W.: J. Math. Phys 46, 032302 (2005)

23. Friedman, J.I., Telegdi, V.L.: Phys. Rev. 105, 1681 (1957)

24. Hughes, V.W., McColm, D.W., Ziock, K., Prepost, R.: Phys. Rev. Lett. 5, 63 (1960)
25. Kuang, Y., et al.: Phys. Rev. A 35, 3172 (1987)

26. Frolov, A.M.: J. Phys. B: At. Mol. Opt. Phys. 37, 2191 (2004)

27. Barham, M., Darewych, J.W.: J. Phys. B Mol. Opt. Phys. 41, 185001 (2008)

28. Frolov, A.M.: J. Phys. B: At. Mol. Opt. Phys. 25, 3059 (1992)

29. Frolov, A.M.: Phys. Rev. A 69, 022501 (2004). Phys. Rev. A 58, 4479 (1998)

30. Mitroy, J., Novikov, S.A.: Phys. Rev. A 70, 032511 (2004)

31. Mezei, Z.S.J., Mitroy, J., Lovas, R.G., Varga, K. Phys. Rev. A 64, 032501 (2001)

32. Richard, J.M. Phys. Rev. A 49, 3573 (1994).

33. Armour, E.A.G., Richard, J.M., Varga, K.: Physics Reports 413, $1-90(2005)$

34. Mitroy, J., Bromley, M.W., Ryzhikh, G.G.: J. Phys. B 35, R81R116 (2002)

35. Suzuki, Y., Varga, K.: Stochastic Variational Approach to Quantum Mechanical Few-Body Problems. Springer-Verlag, Berlin (1998)

36. Kinghorn, D.B., Adamowicz, L.: J. Chem. Phys. 110, 7166 (1999)

37. Bubin, S., Cafiero, M., Adamowicz, L.: J. Chem. Phys. 131, 377 (2003)

38. Bilen'kii, S., van Hieu, N., Nemenov, L., Tkebuchava, F.: Sov J. Nucl. Phys. 10, 469 (1969)

39. Hughes, V.W., Maglic, B.: Bull. Am. Phys. Soc. 16, 65 (1971)

40. A. K Bhatia and R. J. Drachman, Phys Rev A 35, 4051 (1987).

41. Frolov, A.M.: Phys Rev A 69, 022505 (2004)

42. Emami-Razavi, M., Bergeron, N., Darewych, J.W.: Int. J. of Mod. Phys. E 21(11), 1250091 (2012)

43. Gabrielse, G., et al.: Phys. Rev. Lett. 89, 213401 (2002)

44. Amoretti, M., et al.: Nature (London) 419, 456 (2002)

45. Gridnev, D.K., Greiner, C.: Phys. Rev. Lett. 94, 223402 (2005)

46. Banburski, Andrzej, Schuster, Philip: Phys. Rev. D 86, 093007 (2012) 